\title{
Uncovering the Majorana nature through a precision measurement of the $C P$ phase
}

\author{
J. C. Carrasco-Martínez, ${ }^{1,2}$ F. N. Díaz®${ }^{1}{ }^{1}$ and A. M. Gago $\oplus^{1}$ \\ ${ }^{1}$ Sección Física, Departamento de Ciencias, Pontificia Universidad Católica del Perú, \\ Apartado 1761, Lima, Perú \\ ${ }^{2}$ Department of Physics, University of California, Berkeley, California 94720, USA
}

(Received 20 November 2020; accepted 20 January 2022; published 9 February 2022)

\begin{abstract}
We show the possibility to discover the neutrino nature by measuring the Majorana $C P$ phase at the DUNE experiment. This phase is turned on by a decoherence environment, possibly originated by physics at the Planck scale. A sizable distortion in the measurement of the Dirac $C P$ violation phase $\delta_{C P}$ is observed at DUNE when compared with T2HK measurement due to decoherence and the non-null Majorana phase. Being that, when the measurement of the Majorana phase is performed at DUNE, it reaches a precision of 23(21)\% for a decoherence parameter $\Gamma=4.5(5.5) \times 10^{-24} \mathrm{GeV}$ and a Majorana phase equal to $1.5 \pi$. The latter precision is similar to the one obtained at the T2K experiment at its current Dirac $C P$ violation phase measurement.
\end{abstract}

DOI: 10.1103/PhysRevD.105.035010

\section{INTRODUCTION}

The origin of neutrino masses is one of the most relevant questions of modern elementary particle physics $[1,2]$. The Standard Model (SM) Higgs mechanism could generate the neutrino masses if they were Dirac particles. However, this SM alternative does not explain why the neutrino masses are less than one-millionth of the electron's mass, the smallest charged lepton. The general belief is that the latter is resolved through the seesaw mechanism, being the most inexpensive case when neutrinos are Majorana particles [3-15]. The Majorana neutrinos, a fermion that is its own antiparticles, imply the total lepton charge violation, a conserved number within the SM processes [16,17]. Therefore, the quest for elucidating the Majorana nature of neutrinos is one of our best chances to learn about what is beyond the SM. The usual way to look for Majorana neutrinos is using the neutrinoless double beta decay, a lepton charge violating process not allowed by the SM [18-21]. Until now, there is no signal from the latter [22-25] or other ways of proposing searches for Majorana neutrinos [26-29].

The standard oscillation neutrino (SO) probabilities take the same form regardless of the neutrino nature. Only the Dirac $C P$ phase is observable while the two other $C P$ violation phases, when the neutrino is Majorana, are absorbed [30]. However, if we suppose a new physics

Published by the American Physical Society under the terms of the Creative Commons Attribution 4.0 International license. Further distribution of this work must maintain attribution to the author(s) and the published article's title, journal citation, and DOI. Funded by SCOAP ${ }^{3}$.
(NP) phenomenon, subleading to the SO mechanism, exists, the latter situation could be no longer valid. Therefore, this NP could help the Majorana phases to emerge in the oscillation probabilities. The NP phenomena we focus on forecasts an interaction between the neutrino system with the environment at the Planck scale level, which is characterized by a foamy space-time [31]. The loss of quantum coherence (decoherence) in the neutrino system is the interaction's main signature. The foamy space-time is predicted in the context of strings and branes [32-34], and quantum gravity [35]. This quantum decoherence phenomena in the neutrino system have been vastly studied in the literature [36-41] wherein most of the cases contain only the damping effects. Nevertheless, the phenomenology of the interaction between neutrino and a quantum decoherence environment goes beyond the damping effects, being that this might add new contributions to the violation of $C P$ or $C P T$ [42-44]. Through these kinds of contributions in the neutrino oscillation formula, we can reveal the neutrino's Majorana nature. The observability of the Majorana nature using neutrino oscillation is a rather novel approach.

At this point it is worth mentioning that our work's cornerstone hypothesis is that the Dirac $C P$ violation phase measured at $\mathrm{T} 2 \mathrm{~K}$ experiment represents its real value since it is unaffected by quantum decoherence in neutrino oscillations. Thus, considering that the effects of a quantum decoherence environment, coupled with a nonzero Majorana phase, are sizable in the DUNE data, our strategy is divided into two steps. The first step is to assess how much a measurement at the DUNE experiment [45] of the Dirac $C P$ violation phase can deviate from the 
true one. For this purpose, and taking the pure $\mathrm{SO}$ as a theoretical hypothesis in the fitting, the $C P$ violation phase obtained at DUNE is compared with the projected one at the T2HK experiment [46]. The T2HK projected measurement comes to be an upgrade in the precision of the $C P$ violation measurement at $\mathrm{T} 2 \mathrm{~K}$. The second step, and final goal in this paper, is to go beyond testing the DUNE accuracy for determining a Majorana $C P$ phase simultaneously with the decoherence parameter.

\section{GENERAL THEORETICAL FORMALISM}

The treatment for a neutrino subsystem embodied in an infinity unknown reservoir or environment, with which the former interacts weakly, can be obtained by the Lindblad master equation [31]:

$$
\frac{\partial \rho(t)}{\partial t}=-i[H, \rho(t)]+\mathcal{D}[\rho(t)],
$$

where $\rho(t)$ is the reduced (neutrino) density matrix, obtained after trace over the degrees of freedom of the environment, $H$ is the Hamiltonian of the neutrino subsystem and $\mathcal{D}[\rho(t)]$ is the dissipative term which encloses the decoherence phenomena. The aforementioned factor can be written as $\mathcal{D}[\rho(t)]=\frac{1}{2} \sum_{j}\left(\left[A_{j}, \rho(t) A_{j}^{\dagger}\right]+\left[A_{j} \rho(t), A_{j}^{\dagger}\right]\right)$. Thus, if we work in a three-level system the operators $\rho, H$, and $A_{j}$ can be written as follows: $\rho=\sum \rho_{\mu} t_{\mu}, H=\sum h_{\mu} t_{\mu}$, and $A_{j}=\sum a_{\mu}^{j} t_{\mu}$ where $\mu$ is running from 0 to $8, t_{0}$ is the identity matrix and $t_{k}$ the Gell-Mann matrices $(k=1, \ldots, 8)$ that satisfy $\left[t_{a}, t_{b}\right]=i \sum_{c} f_{a b c} t_{c}$, where $f_{a b c}$ are the structure constants of $S U(3)$. The Hermiticity of $\hat{A}_{j}$, which is secured demanding a time-increase Von Neumman entropy, allow us to write a symmetric $\mathbf{D} \equiv D_{k j}$ dissipative/ decoherence matrix as: $D_{k j}=\frac{1}{2} \sum_{l, m, n}\left(a_{n l}\right) f_{k n m} f_{m l j}$, where $a_{n l}=\vec{a}_{n} \cdot \vec{a}_{l} \quad$ with components $\quad D_{\mu 0}=D_{0 \mu}=0$, and $\vec{a}_{r}=\left\{a_{r}^{1}, a_{r}^{2}, \ldots, a_{r}^{8}\right\}$. The matrix $\mathbf{A} \equiv a_{n l}$ should be positive [31] in order to fulfill the complete positivity condition which states that the eigenvalues of the mixing matrix $\rho(t)$ should be positive at any time. Besides, given their inner product structure, the $D_{k j}$ must satisfy the Cauchy-Schwartz inequalities. Adding the conservation of the probability to the aforementioned conditions we get the following evolution equation for $\rho(t)$ :

$$
\dot{\rho}_{0}=0, \quad \dot{\rho}_{k}=\left(H_{k j}+D_{k j}\right) \rho_{j}=M_{k j} \rho_{j},
$$

where $H_{k j}=\sum_{i} h_{i} f_{i j k}$. The matrix form of the solution of Eq. (2) is

$$
\varrho(t)=e^{\mathbf{M} t} \varrho(0)
$$

where $\varrho$ is an eight column vector compose by the $\rho_{k}$ and $\mathbf{M} \equiv M_{k j}$. Hence, the neutrino oscillation probability $\nu_{\alpha} \rightarrow \nu_{\beta}$ is given by:

$$
P_{\nu_{\alpha} \rightarrow \nu_{\beta}}=\frac{1}{3}+\frac{1}{2}\left(\varrho^{\beta}(0)\right)^{T} \varrho^{\alpha}(t)
$$

or written in terms of the coefficients $\rho_{j}^{\alpha}(0)$ :

$$
P_{\nu_{\alpha} \rightarrow \nu_{\beta}}=\frac{1}{3}+\frac{1}{2} \sum_{i, j} \rho_{i}^{\beta}(0) \rho_{j}^{\alpha}(0)\left[e^{\mathbf{M} t}\right]_{i j}
$$

where $\beta, \alpha=e, \mu, \tau$ and $i, j=1, \ldots, 8$.

The coefficients $\rho_{j}^{\alpha}(0)$ and $\rho_{j}^{\beta}(0)$ encloses the elements of the neutrino mixing matrix [47]:

$$
\begin{aligned}
& \rho_{0}^{\alpha}=\sqrt{2 / 3} \\
& \rho_{1}^{\alpha}=2 \operatorname{Re}\left(U_{\alpha 1}^{*} U_{\alpha 2}\right) \\
& \rho_{2}^{\alpha}=-2 \operatorname{Im}\left(U_{\alpha 1}^{*} U_{\alpha 2}\right) \\
& \rho_{3}^{\alpha}=\left|U_{\alpha 1}\right|^{2}-\left|U_{\alpha 2}\right|^{2} \\
& \rho_{4}^{\alpha}=2 \operatorname{Re}\left(U_{\alpha 1}^{*} U_{\alpha 3}\right) \\
& \rho_{5}^{\alpha}=-2 \operatorname{Im}\left(U_{\alpha 1}^{*} U_{\alpha 3}\right) \\
& \rho_{6}^{\alpha}=2 \operatorname{Re}\left(U_{\alpha 2}^{*} U_{\alpha 3}\right) \\
& \rho_{7}^{\alpha}=-2 \operatorname{Im}\left(U_{\alpha 2}^{*} U_{\alpha 3}\right) \\
& \rho_{3}^{\alpha}=\frac{1}{\sqrt{3}}\left(\left|U_{\alpha 1}\right|^{2}+\left|U_{\alpha 2}\right|^{2}-2\left|U_{\alpha 3}\right|^{2}\right)
\end{aligned}
$$

where the $U_{\alpha j}$ are the matrix elements of the PMNS matrix $\left(U_{\text {PMNS }}\right)$ [48] without taking into account the Majorana phases. Thus, in order to include the latter, it is enough to make the replacement:

$$
U_{\text {Majorana }}=U_{\text {PMNS }} \cdot \operatorname{diag}\left(1, e^{-i \phi_{1}}, e^{-i \phi_{2}}\right)
$$

where $\phi_{1}$ and $\phi_{2}$ are the well-known Majorana phases. Therefore, the coefficients takes the following form:

$$
\begin{aligned}
& \rho_{1}^{\alpha} \rightarrow \rho_{1}^{\alpha} \cos \phi_{1}-\rho_{2}^{\alpha} \sin \phi_{1} \\
& \rho_{2}^{\alpha} \rightarrow \rho_{2}^{\alpha} \cos \phi_{1}+\rho_{1}^{\alpha} \sin \phi_{1} \\
& \rho_{3}^{\alpha} \rightarrow \rho_{3}^{\alpha} \\
& \rho_{4}^{\alpha} \rightarrow \rho_{4}^{\alpha} \cos \phi_{2}-\rho_{5}^{\alpha} \sin \phi_{2} \\
& \rho_{5}^{\alpha} \rightarrow \rho_{5}^{\alpha} \cos \phi_{2}+\rho_{4}^{\alpha} \sin \phi_{2} \\
& \rho_{6}^{\alpha} \rightarrow \rho_{6}^{\alpha} \cos \Delta \phi-\rho_{7}^{\alpha} \sin \Delta \phi \\
& \rho_{7}^{\alpha} \rightarrow \rho_{7}^{\alpha} \cos \Delta \phi+\rho_{6}^{\alpha} \sin \Delta \phi \\
& \rho_{8}^{\alpha} \rightarrow \rho_{8}^{\alpha},
\end{aligned}
$$

where $\rho_{j}^{\alpha}$ are the coefficients defined in Eq. (6), which considers only the $U_{\text {PMNS }}$ mixing elements. For other Majorana neutrino mixing matrix parametrizations, the value of the Majorana phases in the above equations can be reinterpreted, see the Appendix A. 


\section{TRANSITION PROBABILITY- PERTURBATIVE APPROACH}

Our selected texture of decoherence matrix $\mathbf{D}$ in the mass vacuum basis (MVB), can be seen as composed by two matrices: one is a diagonal one with its all element equals $\mathbf{D}^{\mathbf{d}}=-\Gamma \times \mathbb{I}$. The other one, $\mathbf{D}^{\mathbf{n d}}$, is composed by its offdiagonal part, having as non-null only a unique $\left[\mathbf{D}^{\mathbf{n d}}\right]_{i j}=$ $-\Gamma_{i j}\left(=-\Gamma_{j i}\right)$ elements (the diagonal is zero). Since in our case, the neutrinos are going to be propagating in matter, and in order to solve the Eq. (2), we need to rotate the decoherence matrix $\mathrm{D}$ to the mass matter basis (MMB). In the MMB the decoherence matrix $\mathbf{D}_{\mathbf{m}}$ is defined as:

$$
\mathbf{D}_{\mathbf{m}}=\mathbf{D}_{\mathbf{m}}^{\mathbf{d}}+\mathbf{D}_{\mathbf{m}}^{\mathbf{n d}},
$$

where $\mathbf{D}_{\mathbf{m}}^{\mathbf{d}}=-\Gamma \times \mathbb{I}$ is purely diagonal, $\mathbf{D}^{\mathbf{d}}$ is unaltered by the rotation, while $\mathbf{D}_{\mathbf{m}}^{\text {nd }}$ is the rotated matrix of the nondiagonal matrix $\mathbf{D}^{\text {nd }}$ in the MVB.

Considering, that the transition probability when neutrinos travel through matter is

$$
P_{\nu_{\alpha} \rightarrow \nu_{\beta}}=\frac{1}{3}+\frac{1}{2}\left(\varrho_{m}^{\beta}(0)\right)^{T} \varrho_{m}^{\alpha}(t)
$$

where $\varrho_{m}^{\alpha}(t)=e^{\mathbf{M} t} \varrho_{m}^{\alpha}(0)$, with $\mathbf{M}=\mathbf{H}_{m}+\mathbf{D}_{m}$. Being $\mathbf{H}_{m}$ the Hamiltonian is written in the MMB. Since $\mathbf{D}_{m}^{d}$ is proportional to the identity matrix, this commutes with $\mathbf{H}_{\mathbf{m}}$, then we have:

$$
\varrho_{m}^{\alpha}(t)=e^{-\Gamma t} e^{\left(\mathbf{H}_{\mathbf{m}}+\mathbf{D}_{m}^{n d}\right) t} \varrho_{m}^{\alpha}(0)=e^{-\Gamma t} \varrho_{m}^{\prime \alpha}(t) .
$$

Given that $\varrho_{m}^{\alpha}(0)=\varrho_{m}^{\prime \alpha}(0)$ we can rewrite the probability in the following way:

$$
P_{\nu_{\alpha} \rightarrow \nu_{\beta}}=\frac{1}{3}+\frac{1}{2} e^{-\Gamma t}\left(\varrho_{m}^{\prime \beta}(0)\right)^{T} \varrho_{m}^{\prime \alpha}(t)
$$

The solution of $\varrho_{m}^{\prime \alpha}(t)$, which is based on a power series solution expanded in $\theta_{13}, \alpha_{\Delta}=\Delta m_{12}^{2} / \Delta m_{13}^{2}$ and a single $\bar{\Gamma}_{i j}=\Gamma_{i j} t$, is shown in the Appendix B. It follows the procedure given in [39].

After assessing the magnitude of the $C P$-odd terms in the transition probability per each one of the off-diagonal elements $\Gamma_{i j}$ (those who activate the Majorana phases), fixed at their maximum absolute allowed values, we conclude that $\Gamma_{28}=-\Gamma / \sqrt{3}$ gives us the most significant deviation from the standard oscillation formulas. The maximum absolute allowed values of $\Gamma_{i j}$ are obtained, individually, through applying the complete positivity conditions [44]. Since all the diagonal elements are equal to $-\Gamma$, the aforementioned maximum values can be written in terms of this singular parameter. In the Appendix $\mathrm{C}$ is displayed the correspondence between each off-diagonal elements, $\Gamma_{i j}$, its ability to turn on $C P$-odd or $C P T$-odd terms and its connection to either $\phi_{1}, \phi_{2}$, or $\Delta \phi=\phi_{1}-\phi_{2}$.

Therefore, taking the off-diagonal part of the decoherence matrix $\mathbf{D}_{\mathbf{n d}}$ formed only with non-null $-\Gamma_{28}$, the following semianalytical perturbative $\nu_{\mu} \rightarrow \nu_{e}$ transition probability formula for SO plus decoherence (DE) is obtained:

$$
\begin{aligned}
P_{\nu_{\mu} \rightarrow \nu_{e}}^{\mathrm{SO}}= & \frac{\left(1-e^{-\bar{\Gamma}}\right)}{3}+P_{\nu_{\mu} \rightarrow \nu_{e}}^{\mathrm{SO}} e^{-\bar{\Gamma}}-\frac{\bar{\Gamma}_{28}}{\sqrt{3}} \sin 2 \theta_{12} \sin ^{2} \theta_{23} \sin \phi_{1} e^{-\bar{\Gamma}} \\
& +\bar{\Gamma}_{28} \theta_{13} \frac{\sin 2 \theta_{23}}{2 \sqrt{3}(A-1) A \Delta}\left(\left(\left(1-A^{2}\right) \cos \delta+A^{2} \cos (\delta-\Delta)-\cos (\delta-A \Delta)\right) \cos \phi_{1}+\left(\left(1-A^{2}\right) \sin \delta\right.\right. \\
& \left.\left.+A^{2} \sin (\delta-\Delta)-\sin (\delta-A \Delta)\right) \cos 2 \theta_{12} \sin \phi_{1}\right) e^{-\bar{\Gamma}}+\bar{\Gamma}_{28} \alpha_{\Delta} \frac{\sin 2 \theta_{12}}{\sqrt{3} A^{2} \Delta}\left(2 \sin ^{2}\left(\frac{A \Delta}{2}\right) \cos 2 \theta_{23} \cos \phi_{1}\right. \\
& \left.-\cos 2 \theta_{12} \sin ^{2} \theta_{23}(\sin A \Delta-A \Delta) \sin \phi_{1}\right) e^{-\bar{\Gamma}}+\cdots
\end{aligned}
$$

where $P_{\nu_{\mu} \rightarrow \nu_{e}}^{\mathrm{SO}}$ is the SO probability in matter $(t \rightarrow L), \Delta=$ $\left(m_{3}^{2}-m_{1}^{2}\right) L /(2 E)$ and $A=\sqrt{2} G_{F} n_{e} L / \Delta$, where $G_{F}$ is the Fermi constant, $n_{e}$ is the electron number density and $L$ is the source-detector distance. The validity of the transition probability formula relies on having the parameterperturbative expansion $\bar{\Gamma}_{28}, \bar{\Gamma}_{28} \theta_{13}$ and $\bar{\Gamma}_{28} \alpha_{\Delta}$ of order: $10^{-2}, 10^{-3}$, and $10^{-4}$, respectively. For instance, the latter values can be attained for $\left|\Gamma_{28}\right|=2 \times 10^{-24} \mathrm{GeV}$, and at DUNE baseline $L=1300 \mathrm{~km}$. Furthermore, to get the antineutrino transition probability is enough to: $\phi_{1} \rightarrow-\phi_{1}, \delta \rightarrow-\delta$ and $A \rightarrow-A$, meanwhile, the $\nu_{\mu}$ survival probability formula is not shown due to its negligible decoherence effect. It is important to point out that $\phi_{2}$ is not considered because it appears in higher-order terms that have negligible contribution in the probability.

The $\nu_{\mu} \rightarrow \nu_{e}$ transition probability displayed in Fig. 1 is numerically calculated at DUNE baseline and for the maximum value of $\Gamma_{28}=-\Gamma / \sqrt{3}$, with $\Gamma=2.5 \times$ $10^{-24} \mathrm{GeV}$ and the following values for the SO parameters, taken from [49]: $\theta_{12}=33.82^{\circ}, \theta_{13}=8.61^{\circ}, \theta_{23}=48.3^{\circ}$, 


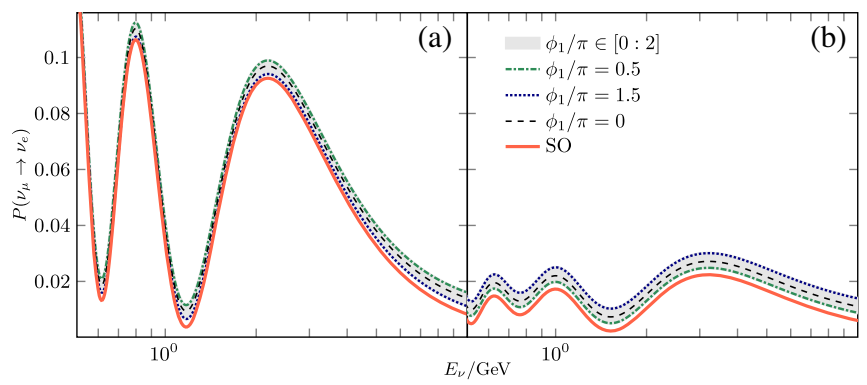

FIG. 1. Oscillation probability depending on the neutrino energy for DUNE experiment. The figures (a) and (b) represent the $\nu_{\mu} \rightarrow \nu_{e}$ and $\bar{\nu}_{\mu} \rightarrow \bar{\nu}_{e}$ appearance channels respectively. The off-diagonal decoherence parameter is $\Gamma_{28}=-\Gamma / \sqrt{3}$. We consider $\Gamma_{28}=-\Gamma / \sqrt{3}, \delta_{C P} / \pi=1.4$, and $\Gamma=2.5 \times 10^{-24} \mathrm{GeV}$.

$\Delta m_{21}^{2}=7.39 \times 10^{-5} \mathrm{eV}^{2}$, and $\Delta m_{31}^{2}=2.523 \times 10^{-3} \mathrm{eV}^{2}$ (normal hierarchy), that are going to be fixed in this paper. The Dirac $C P$ phase is taken as: $\delta_{C P}^{\text {true }} / \pi=1.4$ inspired in the hint given by the T2K experiment [50]. From this figure it is notorious the energy-independent increase of the $\mathrm{SO} \oplus \mathrm{DE}$ probability respect to standard one, regardless the value of $\phi_{1}$, a feature that has been already pointed out in $[39,40]$, for other shape of the decoherence matrix. However, the intensity of this increment depends on $\phi_{1}$, for example, in case of $\phi_{1} / \pi=1.5\left(\phi_{1} / \pi=0.5\right)$ the $\mathrm{SO} \oplus \mathrm{DE}$ neutrino (antineutrino) probability grows much less than its antineutrino (neutrino) counterpart. For $\phi_{1} / \pi=0$ the gain is proportionally the same for both, neutrinos or antineutrinos.

In order to quantify the $C P$ violating effects from the extra terms containing the Majorana phase given in our perturbatives formulas, we use the $C P$ violation asymmetry $\Delta P=P_{\nu_{\mu} \rightarrow \nu_{e}}-P_{\bar{\nu}_{\mu} \rightarrow \bar{\nu}_{e}}$ :

$\Delta P^{\mathrm{SO} \oplus \mathrm{DE}} \simeq \Delta P^{\mathrm{SO}} e^{-\bar{\Gamma}}+\frac{2 \bar{\Gamma}}{3} \sin 2 \theta_{12} \sin ^{2} \theta_{23} \sin \phi_{1} e^{-\bar{\Gamma}}+\cdots$

here it is displayed only the leading term $\bar{\Gamma}_{28} \sim \mathcal{O}(0.01)$ taken $\Gamma_{28}=-\Gamma / \sqrt{3}$, which is its maximum allowed value. The predictions from Eq. (14) are illustrated in Fig. 2 where the $\nu_{\mu} \rightarrow \nu_{e}\left(\bar{\nu}_{\mu} \rightarrow \bar{\nu}_{e}\right)$ transition probability is numerically calculated at DUNE baseline for $\Gamma_{28}=-\Gamma / \sqrt{3}$, with $\Gamma=2.5 \times 10^{-24} \mathrm{GeV}$. In Fig. 2 we see how the overall negative (positive) sign of the decoherence contribution for $\phi_{1} / \pi=1.5\left(\phi_{1} / \pi=0.5\right)$ diminish (increases) the $\Delta P$ amplitude, while for $\phi_{1} / \pi=0.0$ is, as expected, nearly equal to the $\mathrm{SO}$ case.

\section{SIMULATION AND RESULTS}

The DUNE and T2HK simulated data samples are generated with GLoBES [51,52] and nuSQuIDS [53] introducing the configuration and inputs, such as the

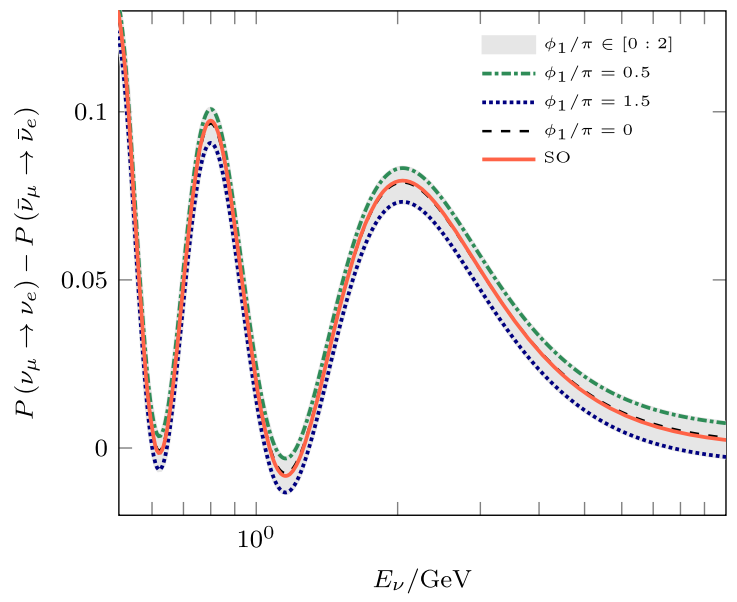

FIG. 2. $\quad C P$ asymmetry depending on the neutrino energy. The off-diagonal decoherence parameter is $\Gamma_{28}=-\Gamma / \sqrt{3}$. We consider $\delta_{C P} / \pi=1.4$, and $\Gamma=2.5 \times 10^{-24} \mathrm{GeV}$.

systematic uncertainties, from $[45,46,54,55]$, and selecting the optimized fluxes for neutrino and antineutrino with 5 years of exposure time per each mode for DUNE with a 40-kt detector. While for T2HK, with 258-kt detector, we consider 3 and 9 years for neutrino and antineutrino mode, respectively. These simulated samples are created for nonnull values of $\Gamma^{\text {true }}$ and $\phi_{1}^{\text {true }}$ and for a value of the Dirac $C P$ violation phase set on the measurement performed by the T2K experiment: $\delta_{C P}^{\text {true }} / \pi=1.4$ [50]. At this point it is important to mention that due to the small statistics and the large size of the uncertainties, we disregard the measurement of the Dirac $C P$ violation claimed by the NOvA experiment, which is $\delta_{C P} / \pi \sim 0.82$ [56]. In this analysis, the $\mathrm{T} 2 \mathrm{~K}$ measurement is considered as the true value of the Dirac $C P$ violation phase since it should be unaltered by any quantum decoherence effects. This is because of the small size of the higher decoherence contributions that would be $\bar{\Gamma} \sim \mathcal{O}(0.001)$, a consequence of combining the source-detector distance of the $\mathrm{T} 2 \mathrm{~K}$ experiment with the $\Gamma$ elected for this study. It should be expected, that the T2HK experiment, with the same source-detector distance, would be also unaffected by the quantum decoherence effects. Within our analysis, the T2HK Dirac $C P$ violation phase simulated measurement, which is an upgrade in the precision of the one performed at $\mathrm{T} 2 \mathrm{~K}$, will be used as a reference point with the expectations at DUNE. The $\chi^{2}$ analysis for DUNE and T2HK relies on the comparison between the SO phenomena, adopted as theoretical hypothesis, and simulated data that incorporates the quantum decoherence effects, where the prescription given in [40,57] is followed. The calculation of the $\Delta \chi^{2}$ is described by:

$$
\begin{aligned}
\Delta \chi^{2}= & \chi^{2}\left(\theta_{13}^{\text {test }}, \delta_{C P}^{\text {test }} ; \theta_{13}^{\text {true }}, \delta_{C P}^{\text {true }}, \Gamma^{\text {true }}, \phi_{1}^{\text {true }}\right) \\
& -\chi_{\min }^{2}\left(\theta_{13}^{\text {fit }}, \delta_{C P}^{\text {fit }} ; \theta_{13}^{\text {true }}, \delta_{C P}^{\text {true }}, \Gamma^{\text {true }}, \phi_{1}^{\text {true }}\right)
\end{aligned}
$$




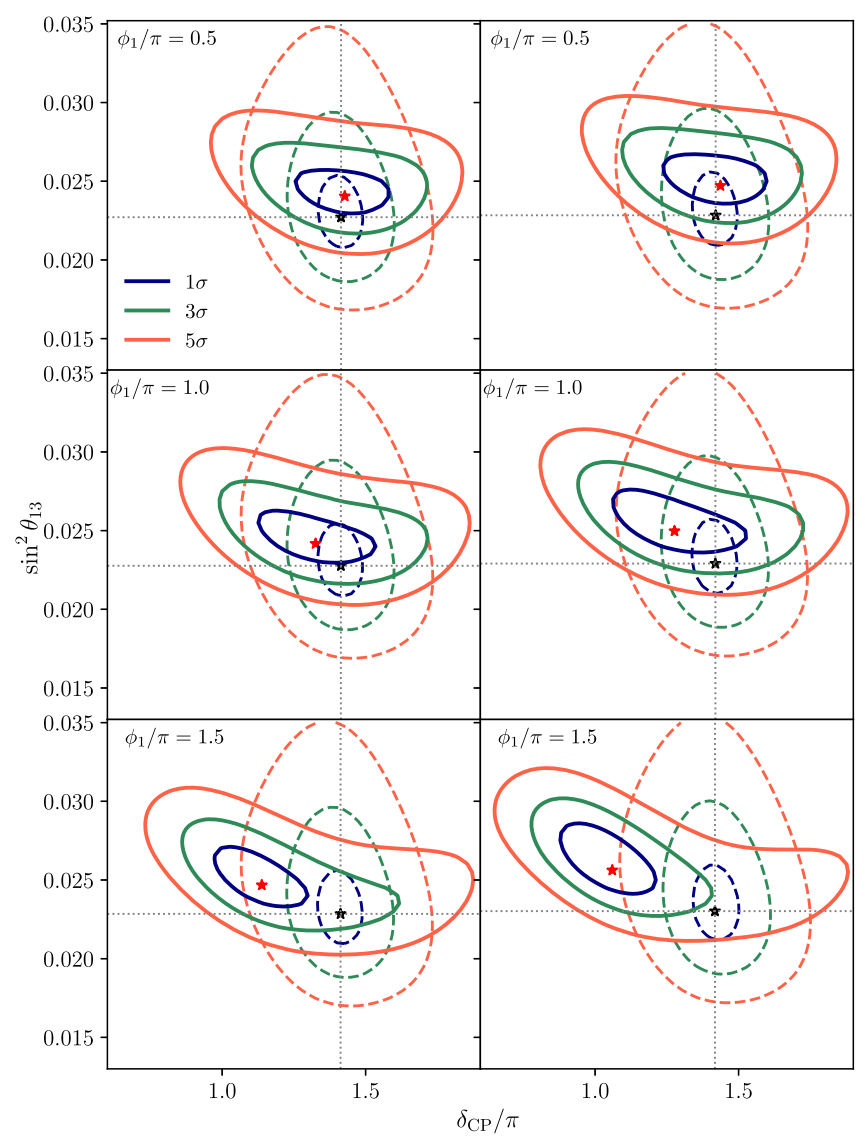

FIG. 3. $\Delta \chi^{2}$ contours $(2$ dof $)$ considering the effects of decoherence with Majorana phases on the standard oscillation fits. The solid and dashed lines are decoherence with $\Gamma_{28}=$ $-\Gamma / \sqrt{3}$ for the DUNE and T2HK experiments, respectively. The left column is $\Gamma=2.5 \times 10^{-24} \mathrm{GeV}$ and the right column is $\Gamma=3.5 \times 10^{-24} \mathrm{GeV}$. We consider $\delta_{C P}^{\text {true }} / \pi=1.4$.

where $\theta_{13}^{\text {fit }}$ and $\delta_{C P}^{\text {fit }}$ are the best-fit points which minimizes the $\chi^{2}$, considering priors at $3 \sigma$ for the rest of the oscillation parameters but $\delta_{C P}$. The DUNE and T2HK $\Delta \chi^{2}$ contours, projected into $\sin ^{2} \theta_{13}$ vs $\delta_{C P}$ planes and obtained after marginalizing over the rest of SO parameters, are presented in Fig. 3. As expected, for T2HK, the $\sin ^{2} \theta_{13}^{\text {fit }}$ and $\delta_{C P}^{\text {fit }}$ are similar to the true ones being unmodified by the parameters chosen for decoherence. Meanwhile, for DUNE there is a slight increase of $\sin ^{2} \theta_{13}^{\text {fit }}$, respect to the $\sin ^{2} \theta_{13}^{\text {true }}(=0.0224)$, explicitly shown in Table I. This increment is the consequence of trying to adjust the theoretical hypothesis, SO, with the energy-independent increase of the $\mathrm{SO} \oplus \mathrm{DE}$ probability amplitude embodied in the data, and modulated by the intensity of $\Gamma$ [see the third term of Eq. (13)].

The $\delta_{C P}^{\text {fit }}$ for DUNE, when $\phi_{1} / \pi=1.5$, is moving away from $\delta_{C P}^{\text {true }} / \pi(=1.4)$ toward $\sim \pi$, minimizing the magnitude of the $C P$ violation asymmetry. For $\phi_{1} / \pi=0.5$ the $\delta_{C P}^{\text {fit }}$ takes almost exactly the value of the true one going in the direction to maximize the $C P$ violation asymmetry. Both features,
TABLE I. Fitted values for $\sin ^{2} \theta_{13}, \delta_{C P}$ and their respective shifts in terms of $\sigma$ units. We consider $\delta_{C P}^{\text {true }} / \pi=1.4$.

\begin{tabular}{lccc}
\hline \hline$\Gamma=2.5 \times 10^{-24} \mathrm{GeV}$ & $\phi_{1} / \pi=0.5$ & $\phi_{1} / \pi=1.0$ & $\phi_{1} / \pi=1.5$ \\
\hline $\sin ^{2} \theta_{13}^{\text {fit }}$ & 0.0241 & 0.0242 & 0.0247 \\
$N_{\sigma}$ & $0.31 \sigma$ & $0.34 \sigma$ & $0.55 \sigma$ \\
$\delta_{C P}^{\text {fit }} / \pi$ & 1.43 & 1.33 & 1.13 \\
$N_{\sigma}$ & $0.08 \sigma$ & $1.19 \sigma$ & $4.34 \sigma$ \\
$\Gamma=3.5 \times 10^{-24} \mathrm{GeV}$ & $\phi_{1} / \pi=0.5$ & $\phi_{1} / \pi=1.0$ & $\phi_{1} / \pi=1.5$ \\
$\sin ^{2} \theta_{13}^{\text {fit }}$ & 0.0247 & 0.0250 & 0.0256 \\
$N_{\sigma}$ & $0.54 \sigma$ & $0.61 \sigma$ & $0.87 \sigma$ \\
$\delta_{C P}^{\text {fit }} / \pi$ & 1.44 & 1.28 & 1.06 \\
$N_{\sigma}$ & $0.14 \sigma$ & $2.37 \sigma$ & $5.47 \sigma$ \\
\hline \hline
\end{tabular}

expressed numerically in Table I, can be explained from the need to accommodate the reduction (increase) of $\Delta P$, when $\phi_{1} / \pi=1.5(0.5)$, seen in Fig. 2. The quantified dislocation, in terms of $\sigma$, from $\sin ^{2} \theta_{13}^{\text {fit }}$ and $\delta_{C P}^{\text {fit }}$ (for DUNE) to the corresponding true ones (for T2HK), for $\Gamma=\{2.5,3.5\} \times$ $10^{-24} \mathrm{GeV}$ and $\phi_{1} / \pi=\{0.0,0.5,1.5\}$, is depicted in Table I. The aforementioned dislocations are estimated by identifying the vertical and horizontal projection of the bestfit point of DUNE on the axes, which center is the T2HK best-fit point (our true point). The vertical corresponds to the $\sin ^{2} \theta_{13}^{\text {fit }}$ dislocation and the horizontal to the $\delta_{C P}^{\mathrm{fit}}$ dislocation, as shown in the Fig. 3. For $\Gamma=3.5(2.5) \times 10^{-24} \mathrm{GeV}$ the most prominent shift is found for $\phi_{1} / \pi=1.5$ with $0.87(0.55) \sigma$ and $5.47(4.34) \sigma$ for $\sin ^{2} \theta_{13}^{\text {fit }}$ and $\delta_{C P}^{\text {fit }}$, respectively. While, for $\Gamma=3.5(2.5) \times 10^{-24} \mathrm{GeV}$, the dislocation of $\delta_{C P}^{\mathrm{fit}}$ reaches $3 \sigma(2 \sigma)$ and $5 \sigma(3 \sigma)$ when $\phi_{1} / \pi$ takes values below 1.01 (1.03) and $1.30(1.10)$, respectively, the $\sin ^{2} \theta_{13}^{\text {fit }}$ is clearly stable in front of changes along the $\phi_{1}$ interval. The less significant distortion is for $\phi_{1} / \pi=0.5$ with $0.54(0.31) \sigma$ and $0.14(0.08) \sigma$ for $\sin ^{2} \theta_{13}^{\mathrm{fit}}$ and $\delta_{C P}^{\mathrm{fit}}$, respectively. Our approach has been to assess, separately, the distortion of $\delta_{C P}^{\text {fit }}$ and $\sin ^{2} \theta_{13}^{\text {fit }}$ with respect to the $\delta_{C P}^{\text {true }}$ and $\sin ^{2} \theta_{13}^{\text {true }}$, respectively. Through this framework, we can make evident a very sizable distortion on $\delta_{C P}$. It is clear that if we use an analysis like the PG-test [58], which measures the compatibility between data sets, the global discrepancy is going to be lower than the isolated one for $\delta_{C P}$. In fact, we obtain $3.4 \sigma$ of discrepancy between DUNE and T2HK for $\Gamma=$ $3.5 \times 10^{-24} \mathrm{GeV}$ and $\phi_{1} / \pi=1.5$.

On the other hand, a way to discriminate between different values of $\phi_{1}$ is through the ratio $(\mathcal{R})$ of the number of $\sigma$ deviation for $\sin ^{2} \theta_{13}^{\text {fit }}$ to the corresponding ones for $\delta_{C P}^{\text {fit }}$. In fact, a sort of discernment is achieved, for instance, for $\Gamma=3.5(2.5) \times 10^{-24} \mathrm{GeV}, \mathcal{R} \sim 0.16(0.13)-0.26(0.29)$ for the interval $\phi_{1} / \pi=1.0,1.5$ reaching values up to $\sim 3.86(3.88)$ for $\phi_{1} / \pi=0.5$. A plus that reinforces the utility of $\mathcal{R}$ it is its low variations against changes of $\Gamma$. 


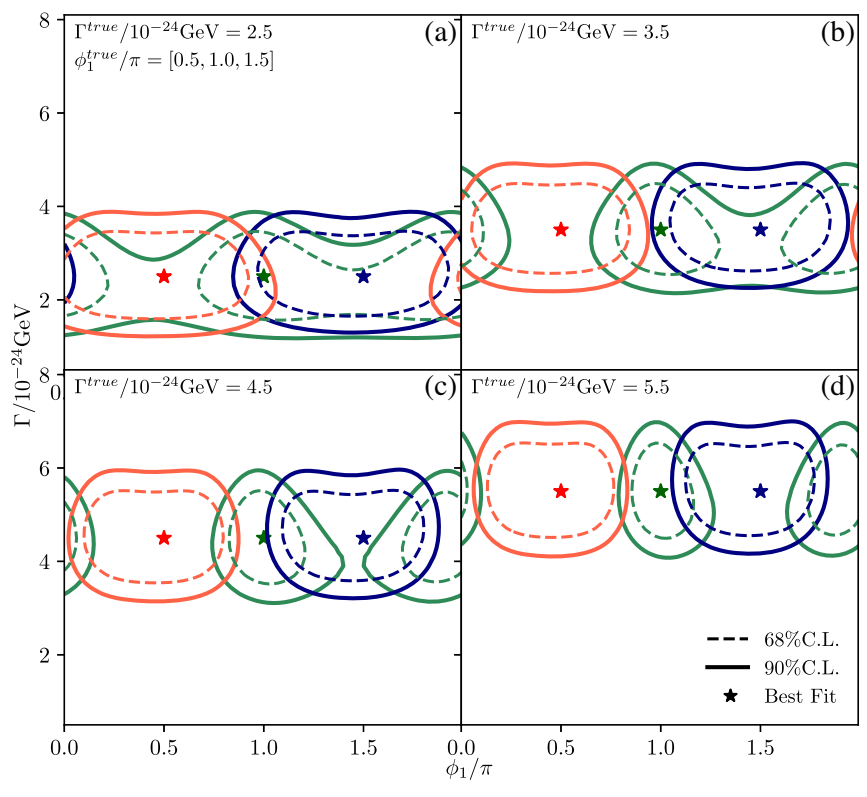

FIG. 4. DUNE's ability to constrain the decoherence parameter and the Majorana phase. The figures (a), (b), (c) and (d) show $\Gamma^{\text {true }} / 10^{-24} \mathrm{GeV}=2.5,3.5,4.5$ and 5.5 , accordingly. The dashed and solid lines represent the 68\% C.L. and 90\% C.L. for 2 dof, while the red, green, and blue lines represent $\phi^{\text {true }} / \pi=0.5,1.0,1.5$, respectively.

The aforementioned analysis had the purpose of searching for distortions in $\sin ^{2} \theta_{13}^{\mathrm{fit}}$ and $\delta_{C P}^{\mathrm{fit}}$, considering pure SO as a theoretical hypothesis. Now, the aim is to go one step further and to explore the capacity of DUNE for measuring the Majorana phase, and also $\Gamma$, under the (SO) plus decoherence (DE) as theoretical hypothesis, for $\phi_{1} / \pi=0.5,1$ and 1.5. We use for $\Gamma / 10^{-24} \mathrm{GeV}=2.5$, 3.5, 4.5, and 5.5. In Fig. 4 the different allowed regions are displayed considering $68 \%$ and $90 \%$ C.L. for 2 dof. For $\Gamma / 10^{-24} \mathrm{GeV}=2.5$ and 3.5 is not possible to clearly disentangle the value of $\phi_{1} / \pi=1.0$ at $90 \%$ of C.L. from $\phi_{1} / \pi=0.5$ and 1.5. Meanwhile, for slightly increased values of $\Gamma / 10^{-24} \mathrm{GeV}=4.5$ and 5.5 , the value of $\phi_{1} / \pi=$ 1.0 is excluded at $90 \%$ either for $\phi_{1} / \pi=0.5$ and 1.5 , being able to separate between the chosen values of the Majorana phases $\phi_{1}$. Thus, it is seen that DUNE is able to measure $\phi_{1} / \pi=1.50 \pm 0.35(0.32)$ and $\phi_{1} / \pi=0.50 \pm 0.35(0.32)$ and $\Gamma=4.50 \pm 1.38(5.50 \pm 1.42) \times 10^{-24} \mathrm{GeV}$. While for $\phi_{1} / \pi=1.0 \pm 0.19(0.15)$ a $\Gamma=4.50 \pm 1.42(5.50 \pm$ $1.46) \times 10^{-24} \mathrm{GeV}$ is obtained.

All the values of $\Gamma$ used in our analysis are below the decoherence limits for handmade sources [41] and cannot be compared with the limits imposed by Ice Cube [59] since we are considering a nondiagonal scenario for the decoherence matrix.

\section{SUMMARY AND CONCLUSIONS}

Assuming the existence of the decoherence environment, probably caused by Planck scale physics, we demonstrated the possibility of uncovering the Majorana nature of neutrinos in the DUNE experiment. Our approach is at first to show the strong displacement that it would be exhibited by the measured value of $\delta_{C P}$ at DUNE, in comparison with the one measured at T2HK, which would be unaffected by the decoherence effects. For a decoherence parameter $\Gamma=3.5 \times 10^{-24} \mathrm{GeV}$, the aforementioned displacement can be as large as $5.47 \sigma$ for a Majorana phase $\phi_{1} / \pi=1.5$. Next, we assessed the power of DUNE experiment in constraining the Majorana phase achieving, for instance, a precision of $23 \%$ (21\%) for $\phi_{1} / \pi=1.5$ with $\Gamma=$ $4.5(5.5) \times 10^{-24} \mathrm{GeV}$. These values of precision are compatible with the current results on the Dirac $C P$ phase reached by the T2K experiment [50]. Finally, we can conclude that, if decoherence exists in the manner is predicted here, there would be an interesting chance for DUNE to perform a first time a measurement of the Majorana $C P$ phase, with some reasonable uncertainties.

\section{ACKNOWLEDGMENTS}

A. M. G. acknowledges funding by the Dirección de Gestión de la Investigación at PUCP, through grants No. DGI-2017-3-0019 and No. DGI 2019-3-0044. F. N.D. acknowledges CONCYTEC for the graduate fellowship under Grant No. 236-2015-FONDECYT. The authors also want to thank F. de Zela, J. Jones-Pérez, J. L. Bazo, C. A. Argüelles, and Mario A. Acero for useful suggestions and reading the manuscript.

\section{APPENDIX A: OTHER PARAMETRIZATIONS OF THE MAJORANA NEUTRINO MIXING MATRIX}

\section{Symmetrical parametrization of the mixing matrix}

The elements of symmetric parametrization of the mixing matrix, given in Eq. (5) in [60], assuming the relation $\delta=\phi_{13}-\phi_{12}-\phi_{23}$, can be written as follows:

$$
\begin{aligned}
U_{e 1} & \rightarrow U_{e 1} \\
U_{e 2} & \rightarrow U_{e 2} e^{-i \phi_{12}} \\
U_{e 3} & \rightarrow U_{e 3} e^{-i\left(\phi_{23}+\phi_{12}\right)} \\
U_{\mu 1} & \rightarrow U_{\mu 1} e^{i \phi_{12}} \\
U_{\mu 2} & \rightarrow U_{\mu 2} \\
U_{\mu 3} & \rightarrow U_{\mu 3} e^{-i \phi_{23}} \\
U_{\tau 1} & \rightarrow U_{\tau 1} e^{i\left(\phi_{23}+\phi_{12}\right)} \\
U_{\tau 2} & \rightarrow U_{\tau 2} e^{i \phi_{23}} \\
U_{\tau 3} & \rightarrow U_{\tau 3}
\end{aligned}
$$

where $\phi_{13}, \phi_{12}$, and $\phi_{23}$ are the $C P$ phases used in [60].

The corresponding $\rho_{j}^{\alpha}$ are described by the following relations: 
TABLE II. Parametrizations comparison.

\begin{tabular}{lcc}
\hline \hline Sym $\leftrightarrow$ PDG I & Sym $\leftrightarrow$ Our Work & PDG I $\leftrightarrow$ Our Work \\
\hline$\phi_{12}+\phi_{23} \leftrightarrow \phi_{1}$ & $\phi_{12} \leftrightarrow \phi_{1}$ & $\phi_{1} \leftrightarrow \Delta \phi$ \\
$\phi_{23} \leftrightarrow \phi_{2}$ & $\phi_{12}+\phi_{23} \leftrightarrow \phi_{2}$ & $\phi_{2} \leftrightarrow \phi_{1}$ \\
$\phi_{12} \leftrightarrow \Delta \phi$ & $\phi_{23} \leftrightarrow-\Delta \phi$ & $\Delta \phi \leftrightarrow-\phi_{2}$ \\
\hline \hline
\end{tabular}

$$
\begin{aligned}
& \rho_{1}^{\alpha} \rightarrow \rho_{1}^{\alpha} \cos \phi_{12}-\rho_{2}^{\alpha} \sin \phi_{12} \\
& \rho_{2}^{\alpha}=\rho_{2}^{\alpha} \cos \phi_{12}+\rho_{1}^{\alpha} \sin \phi_{12} \\
& \rho_{3}^{\alpha} \rightarrow \rho_{3}^{\alpha} \\
& \rho_{4}^{\alpha} \rightarrow \rho_{4}^{\alpha} \cos \left(\phi_{12}+\phi_{23}\right)-\rho_{5}^{\alpha} \sin \left(\phi_{12}+\phi_{23}\right) \\
& \rho_{5}^{\alpha} \rightarrow \rho_{5}^{\alpha} \cos \left(\phi_{12}+\phi_{23}\right)+\rho_{4}^{\alpha} \sin \left(\phi_{12}+\phi_{23}\right) \\
& \rho_{6}^{\alpha} \rightarrow \rho_{6}^{\alpha} \cos \phi_{23}-\rho_{7}^{\alpha} \sin \phi_{23} \\
& \rho_{7}^{\alpha} \rightarrow \rho_{7}^{\alpha} \cos \phi_{23}+\rho_{7}^{\alpha} \sin \phi_{23} \\
& \rho_{8}^{\alpha} \rightarrow \rho_{8}^{\alpha},
\end{aligned}
$$

where $\rho_{j}^{\alpha}$ are given in Eq. (6)

\section{Particle data group parametrization type I: PDG I}

Here we analyze the mixing matrix parametrization given in [61], which includes the Majorana phases $\phi_{1}$ and $\phi_{2}$ :

$$
U_{\text {Majorana }}=U_{\text {PMNS }} \cdot \operatorname{diag}\left(\exp i \phi_{1}, \exp i \phi_{2}, 1\right)
$$

The corresponding $\rho_{j}^{\alpha}$ are described by the following equations:

$$
\begin{aligned}
& \rho_{1}^{\alpha} \rightarrow \rho_{1}^{\alpha} \cos \Delta \phi+\rho_{2}^{\alpha} \sin \Delta \phi \\
& \rho_{2}^{\alpha} \rightarrow \rho_{2}^{\alpha} \cos \Delta \phi-\rho_{1}^{\alpha} \sin \Delta \phi \\
& \rho_{3}^{\alpha} \rightarrow \rho_{3}^{\alpha} \\
& \rho_{4}^{\alpha} \rightarrow \rho_{4}^{\alpha} \cos \phi_{1}-\rho_{5}^{\alpha} \sin \phi_{1} \\
& \rho_{5}^{\alpha} \rightarrow \rho_{5}^{\alpha} \cos \phi_{1}+\rho_{4}^{\alpha} \sin \phi_{1} \\
& \rho_{6}^{\alpha} \rightarrow \rho_{6}^{\alpha} \cos \phi_{2}-\rho_{7}^{\alpha} \sin \phi_{2} \\
& \rho_{7}^{\alpha} \rightarrow \rho_{7}^{\alpha} \cos \phi_{2}+\rho_{6}^{\alpha} \sin \phi_{2} \\
& \rho_{8}^{\alpha} \rightarrow \rho_{8}^{\alpha},
\end{aligned}
$$

Below, we present in Table II a summary of the equivalences between the different parametrizations.

where $\Delta \phi=\phi_{1}-\phi_{2}$.

\section{APPENDIX B: PROBABILITY CALCULATION}

For solving $\varrho_{m}^{\prime \alpha}(t)$ we must start with the next differential equation:

$$
\stackrel{i \alpha}{\varrho_{m}^{\alpha}}=\left(\mathbf{H}_{\mathbf{m}}+\mathbf{D}_{m}^{n d}\right) \varrho_{m}^{\prime \alpha}
$$

which is similar to Eq. (2) presented in our letter. Before continue, we must point out that the following procedure is similar to the one given in [39]. The Eq. (B1) can be simplified using this change of variable:

$$
\varrho_{m}^{\alpha}(t)=e^{\mathbf{H}_{\mathrm{m}} t} \widetilde{Q}^{\alpha}(t)
$$

then, the Eq. (B1):

$$
e^{\mathbf{H}_{\mathbf{m}}} \dot{\tilde{Q}}^{\alpha}+\mathbf{H}_{m} e^{\mathbf{H}_{\mathbf{m}} t} \tilde{Q}^{\alpha}=\left(\mathbf{H}_{\mathbf{m}}+\mathbf{D}_{\mathbf{m}}^{\mathbf{n d}}\right) e^{\mathbf{H}_{\mathbf{m}} t} \tilde{\varrho}^{\alpha}
$$

thus we get:

$$
\dot{\tilde{Q}}^{\alpha}=e^{-\mathbf{H}_{\mathbf{m}} t} \mathbf{D}_{\mathbf{m}}^{\mathbf{n d}} e^{-\mathbf{H}_{\mathbf{m}} t} \tilde{Q}^{\alpha},
$$

the matrix $e^{-\mathbf{H}_{\mathbf{m}} t} \mathbf{D}_{\mathbf{m}}^{\mathbf{n d}} e^{-\mathbf{H}_{\mathbf{m}} t}$ can be expanded perturbatively in power series of the small parameters $\theta_{13}$, and $\alpha_{\Delta}$ which turns out to be

$$
e^{-\mathbf{H}_{\mathbf{m}} t} \mathbf{D}_{\mathbf{m}}^{\mathbf{n d}} e^{-\mathbf{H}_{\mathbf{m}} t}=\Gamma_{i j}\left(\tilde{D}^{(0)}+\theta_{13} \tilde{D}^{\left(\theta_{13}\right)}+\alpha_{\Delta} \tilde{D}^{\left(\alpha_{\Delta}\right)}+\cdots\right)
$$

we can factor out the decoherence parameter $\Gamma_{i j}$ since it is a common factor of all the elements in the decoherence matrix $\mathbf{D}_{\mathbf{m}}^{\text {nd }}$ in the MMB (a consequence of its definition in the mass vacuum basis that is an off-diagonal matrix with only non-null terms in a given the $-\Gamma_{i j}$ element). Replacing Eq. (B5) into Eq. (B4):

$$
\dot{\tilde{\varrho}}^{\alpha}=\Gamma_{i j}\left(\tilde{D}^{(0)}+\theta_{13} \tilde{D}^{\left(\theta_{13}\right)}+\alpha_{\Delta} \tilde{D}^{\left(\alpha_{\Delta}\right)}+\cdots\right) \tilde{\varrho}^{\alpha}
$$

the above equation can be solved perturbatively treating $\tilde{\varrho}^{\alpha}$ as a power series in $\theta_{13}, \alpha_{\Delta}$ and $\Gamma_{i j}$ :

$$
\begin{aligned}
\tilde{\varrho}^{\alpha}= & \tilde{\varrho}^{(0)}+\theta_{13} \tilde{\varrho}^{(\theta)}+\alpha_{\Delta} \tilde{\varrho}^{\left(\alpha_{\Delta}\right)}+\alpha_{\Delta} \theta_{13} \tilde{\varrho}^{\left(\alpha_{\Delta} \theta_{13}\right)}+\cdots \\
& +\Gamma_{i j} \tilde{\varrho}^{\left(\Gamma_{i j}\right)}+\Gamma_{i j} \theta_{13} \tilde{\varrho}^{\left(\Gamma_{i j} \theta_{13}\right)}+\Gamma_{i j} \alpha_{\Delta} \tilde{\varrho}^{\left(\Gamma_{i j} \alpha_{\Delta}\right)}+\cdots
\end{aligned}
$$

Then substituing Eq. (B7) into Eq. (B6) we produce a sequence of first order differential equations each of them collecting equal power terms. The $\Gamma_{i j}$-independent terms of the $\tilde{\varrho}^{\alpha}$ expansion: $\tilde{\varrho}^{(0)}+\theta_{13} \tilde{\varrho}^{(\theta)}+\alpha_{\Delta} \tilde{\varrho}^{\left(\alpha_{\Delta}\right)}+$ $\alpha_{\Delta} \theta_{13} \tilde{\varrho}^{\left(\alpha_{\Delta} \theta_{13}\right)}+\cdots$ corresponds to the initial condition $\tilde{\varrho}^{\alpha}(0)$, which is constant in time and coincides with the initial condition for the standard oscillation case, since at that instant the environment is decoupled (not interacting) with the neutrino system. Considering all the latter plus the condition that $\varrho_{m}^{\prime \alpha}(0)=\tilde{\varrho}^{\alpha}(0)$, we can rewrite Eq. (B2) as follows:

$$
\varrho_{m}^{\prime \alpha}(t)=e^{\mathbf{H}_{\mathbf{m}} t}\left(\varrho_{m}^{\prime \alpha}(0)+\bar{\Gamma}_{i j}(\ldots)\right) .
$$


with $\bar{\Gamma}_{i j}=\Gamma_{i j} t$. The second term on the right-hand side of the equation above contains the explicit solution of the power series of $\varrho^{\alpha}$.

\section{APPENDIX C: $C P$-ODD, $C P T$-ODD TERMS AND MAJORANA PHASES}

In Table III we present a classification of the correspondence between each one of the off-diagonal elements $\Gamma_{i j}$ and $\phi_{1}, \phi_{2}$, or $\Delta \phi$, also pointing out its connection with $C P$-odd, $C P T$-odd terms or both in the oscillation probabilities which incorporates quantum decoherence.
TABLE III. Violation of symmetries by nondiagonal decoherence elements and their dependence on the Majorana phases.

\begin{tabular}{lcc}
\hline \hline $\mathrm{CPV}$ & $\mathrm{CPTV}$ & $\begin{array}{c}\text { Non-null Majorana } \\
\text { phase }\end{array}$ \\
\hline$\Gamma_{13}, \Gamma_{23}, \Gamma_{18}, \Gamma_{28}, \Gamma_{12}$ & $\Gamma_{23}, \Gamma_{28}, \Gamma_{12}$ & $\phi_{1}$ \\
$\Gamma_{34}, \Gamma_{35}, \Gamma_{48}, \Gamma_{58}, \Gamma_{45}$ & $\Gamma_{35}, \Gamma_{58}, \Gamma_{45}$ & $\phi_{2}$ \\
$\Gamma_{37}, \Gamma_{36}, \Gamma_{68}, \Gamma_{67}, \Gamma_{78}$ & $\Gamma_{37}, \Gamma_{67}, \Gamma_{78}$ & $\Delta \phi$ \\
$\Gamma_{14}, \Gamma_{24}, \Gamma_{15}, \Gamma_{25}$ & $\Gamma_{24}, \Gamma_{15}$ & $\phi_{1}, \phi_{2}$ \\
$\Gamma_{16}, \Gamma_{17}, \Gamma_{26}, \Gamma_{27}$ & $\Gamma_{17}, \Gamma_{26}$ & $\phi_{1}, \Delta \phi$ \\
$\Gamma_{46}, \Gamma_{47}, \Gamma_{56}, \Gamma_{57}$ & $\Gamma_{47}, \Gamma_{56}$ & $\phi_{2}, \Delta \phi$ \\
\hline \hline
\end{tabular}

[1] H. Murayama, Prog. Part. Nucl. Phys. 57, 3 (2006).

[2] J. W. F. Valle, Adv. Ser. Dir. High Energy Phys. 25, 25 (2015).

[3] T. Yanagida, Conf. Proc. C 7902131, 95 (1979).

[4] M. Gell-Mann, P. Ramond, and R. Slansky, Conf. Proc. C 790927, 315 (1979).

[5] S. L. Glashow, NATO Sci. Ser. B 61, 687 (1980).

[6] P. Minkowski, Phys. Lett. 67B, 421 (1977).

[7] R. N. Mohapatra and G. Senjanovic, Phys. Rev. Lett. 44, 912 (1980).

[8] M. Magg and C. Wetterich, Phys. Lett. 94B, 61 (1980).

[9] J. Schechter and J. W. F. Valle, Phys. Rev. D 22, 2227 (1980).

[10] R. N. Mohapatra and G. Senjanovic, Phys. Rev. D 23, 165 (1981).

[11] R. Foot, H. Lew, X. G. He, and G. C. Joshi, Z. Phys. C 44, 441 (1989).

[12] R. E. Shrock, Phys. Rev. D 24, 1232 (1981).

[13] W. Konetschny and W. Kummer, Phys. Lett. 70B, 433 (1977).

[14] T. P. Cheng and L.F. Li, Phys. Rev. D 22, 2860 (1980).

[15] G. Lazarides, Q. Shafi, and C. Wetterich, Nucl. Phys. B181, 287 (1981).

[16] A. Atre, T. Han, S. Pascoli, and B. Zhang, J. High Energy Phys. 05 (2009) 030.

[17] A. Zee, Nucl. Phys. B264, 99 (1986).

[18] S. M. Bilenky and C. Giunti, Mod. Phys. Lett. A 27, 1230015 (2012).

[19] A. Faessler and F. Simkovic, J. Phys. G 24, 2139 (1998).

[20] F. T. Avignone, S. R. Elliott, and J. Engel, Rev. Mod. Phys. 80, 481 (2008).

[21] J. D. Vergados, H. Ejiri, and F. Simkovic, Rep. Prog. Phys. 75, 106301 (2012).

[22] M. Agostini et al. (GERDA Collaboration), Science 365, 1445 (2019).

[23] G. Anton et al. (EXO-200 Collaboration), Phys. Rev. Lett. 123, 161802 (2019).

[24] D. Q. Adams et al. (CUORE Collaboration), Phys. Rev. Lett. 124, 122501 (2020).
[25] A. Gando, Y. Gando, T. Hachiya, A. Hayashi, S. Hayashida, H. Ikeda et al. (KamLAND-Zen Collaboration), Phys. Rev. Lett. 117, 082503 (2016).

[26] A. M. Sirunyan et al. (CMS Collaboration), J. High Energy Phys. 01 (2019) 122.

[27] R. Aaij et al. (LHCb Collaboration), Phys. Rev. Lett. 112, 131802 (2014).

[28] A. B. Balantekin, A. de Gouvêa, and B. Kayser, Phys. Lett. B 789, 488 (2019).

[29] K. Bora, D. Borah, and D. Dutta, Phys. Rev. D 96, 075006 (2017).

[30] C. Giunti, Phys. Lett. B 686, 41 (2010).

[31] F. Benatti and R. Floreanini, J. High Energy Phys. 02 (2000) 032; Phys. Rev. D 64, 085015 (2001).

[32] J. R. Ellis, N. E. Mavromatos, and D. V. Nanopoulos, Phys. Lett. B 293, 37 (1992).

[33] J. R. Ellis, N. E. Mavromatos, and D. V. Nanopoulos, Int. J. Mod. Phys. A 11, 1489 (1996).

[34] F. Benatti and R. Floreanini, Ann. Phys. (N.Y.) 273, 58 (1999).

[35] S. Hawking, Commun. Math. Phys. 87, 395 (1982); Phys. Rev. D 37, 904 (1988); Phys. Rev. D 53, 3099 (1996); S. W. Hawking and C. J. Hunter, Phys. Rev. D 59, 044025 (1999).

[36] E. Lisi, A. Marrone, and D. Montanino, Phys. Rev. Lett. 85, 1166 (2000).

[37] G. Barenboim, N. E. Mavromatos, S. Sarkar, and A. Waldron-Lauda, Nucl. Phys. B758, 90 (2006).

[38] P. Bakhti, Y. Farzan, and T. Schwetz, J. High Energy Phys. 05 (2015) 007.

[39] J. A. Carpio, E. Massoni, and A. M. Gago, Phys. Rev. D 97, 115017 (2018).

[40] J. A. Carpio, E. Massoni, and A. M. Gago, Phys. Rev. D 100, 015035 (2019).

[41] A. L. G. Gomes, R. A. Gomes, and O. L. G. Peres, arXiv: 2001.09250.

[42] R. L. N. Oliveira, M. M. Guzzo, and P. C. de Holanda, Phys. Rev. D 89, 053002 (2014).

[43] A. Capolupo, S. M. Giampaolo, and G. Lambiase, Phys. Lett. B 792, 298 (2019).

[44] J. C. Carrasco, F. N. Díaz, and A. M. Gago, Phys. Rev. D 99, 075022 (2019).

[45] R. Acciarri et al. (DUNE Collaboration), arXiv:1512.06148. 
[46] K. Abe et al. (Hyper-Kamiokande Collaboration), arXiv: 1805.04163.

[47] A. M. Gago, E. M. Santos, W. J. C. Teves, and R. Z. Funchal, arXiv:hep-ph/0208166.

[48] Z. Maki, M. Nakagawa, and S. Sakata, Prog. Theor. Phys. 28, 870 (1962).

[49] http://www.nu-fit.org.

[50] K. Abe et al. (T2K Collaboration), Nature (London) 580, 339 (2020).

[51] P. Huber, M. Lindner, and W. Winter, Comput. Phys. Commun. 167, 195 (2005).

[52] P. Huber, J. Kopp, M. Lindner, M. Rolinec, and W. Winter, Comput. Phys. Commun. 177, 432 (2007).

[53] C. A. A. Delgado, J. Salvado, and C. N. Weaver, Comput. Phys. Commun. 196, 569 (2015).
[54] T. Alion et al. (DUNE Collaboration), arXiv:1606.09550.

[55] P. Huber, M. Mezzetto, and T. Schwetz, J. High Energy Phys. 03 (2008) 021.

[56] A. Himmel, New oscillation results from the NOvA experiment—presented at Neutrino2020, 2020.

[57] F. N. Díaz, J. Hoefken, and A. M. Gago, Phys. Rev. D 102, 055020 (2020).

[58] M. Maltoni and T. Schwetz, Phys. Rev. D 68, 033020 (2003).

[59] P. Coloma, J. Lopez-Pavon, I. Martinez-Soler, and H. Nunokawa, Eur. Phys. J. C 78, 614 (2018).

[60] W. Rodejohann and J. W. F. Valle, Phys. Rev. D 84, 073011 (2011).

[61] M. Tanabashi et al. (Particle Data Group), Phys. Rev. D 98, 030001 (2018). 